\title{
Feasibility analysis of the satellite-based wide area differential GPS application in ocean survey
}

\author{
Shao Mingqin \\ Maritime College of Shandong Jiaotong University Weihai, China
}

\begin{abstract}
Keywords: satellite-based wide area differential GPS, positioning accuracy, ocean water depth survey, application
\end{abstract}

\begin{abstract}
In order to obtain more accurate data of the ocean water depth, this paper is based on the principle of satellite-based wide area differential GPS positioning technology, through a test, positioning accuracy of differential RTK, differential beacon and satellite-based wide area differential GPS are compared and analyzed. This test shows that the satellite-based wide area differential GPS positioning technology in ocean water depth survey can meet the accuracy requirements, so this technology can make ocean water depth survey more convenient.
\end{abstract}

\section{Introduction}

GPS single point real-time positioning accuracy can only reach about $10 \mathrm{~m}$ [1]. In order to further improve the positioning accuracy, differential GPS positioning method emerge as the times require. With the development of differential GPS technology, differential GPS can effectively improve the GPS real-time positioning accuracy, broadens the scope of its application. According to the different principle of error separation, differential GPS can be divided into local area differential GPS and wide area differential GPS. In the local area differential GPS, the positioning points are usually at a distance which is closer to the reference station, observation error has correlation. The biggest disadvantage of this method lies in the effectiveness of differential correction reduced rapidly with the increasing distance of the receiver and the reference station. Wide area differential GPS (WADGPS) focuses on the analysis of error sources and further makes the measurement error model.

There are provisions in $<$ Specifications for hydrographic survey $>[2]$, in the process of ocean water depth survey, the positioning error in map should be not greater than $1.0 \mathrm{~mm}$, when using less than (including) 1: 5000 scale mapping and more than (including) 1: 100000 scale mapping. When we carry out the water depth survey in the sea area near the coast, we often use the beacon differential mode to make a position, but when we carry out the water depth survey in the ocean area and in the offshore waters which lies in more than 200 nautical miles from shore, because of the coverage range of local area differential GPS is limited, we often use the GPS single point positioning. When using more than (including) 1: 25000 scale mapping, if we use the traditional way of positioning, positioning accuracy can not meet the requirements of specification, satellite-based wide area difference GPS can solve this problem, the real-time differential GPS positioning accuracy can meet the requirements. This paper shows that it is feasible to carry out large scale water depth survey in the ocean area by using this model.

\section{The positioning principle of satellite-based wide area differential GPS and MSAS system}

\section{The positioning principle of satellite-based wide area differential GPS [3]}

Research shows that broadcast ephemeris error, satellite clock error and influence of ionospheric activity on Sunday are the main cause of the real-time positioning accuracy is not high. WADGPS can use the reference stations which are distributed all over the world to solve all the above errors in real-time. The technical principle of WADGPS is: using a master station and a plurality of secondary stations which are set up in known coordinate points to observe the satellite synchronously, the received signal is transmitted to the main station in real time, the main station can distinguish and model the error sources of GPS measurements and calculate the correction 
value (differential value) for every error sources, then transmit the correction data to the user through the data communication chain in real time, the user can use these correction data to correct the error in GPS positioning and to achieve the purpose of weakening these error sources and improving the positioning accuracy of GPS.

In the WADGPS system, the main problem is the data communication, its difficulty lies in two aspects: the first one is the correct signal must have large enough coverage range, the second one is the receiving device of user station must be portable and cheap. However, the satellite-based wide area differential GPS uses a number of geostationary satellite, provides the ranging signals which are similar to the L1 and further enhances the wide area differential GPS. At present, the SBAS system of the global development is: European Space Agency satellite navigation system, it can cover Europe continent. Wide area augmentation system of the United States of America, it can cover American continent. Multi-function satellite augmentation system of Japan (MSAS), it can cover Asia continent. These three systems are fully compatible with interoperability.

\section{The composition of MSAS system and its coverage range}

The MSAS of Japan is GPS satellite-based augmentation system based on two multi-function satellites (MTSAT), its space constellation consists of two multi-function satellites (MTSAT). MTSAT is a geostationary satellite (GEO), its fixed position is in east longitude 140 degrees and 145 degrees respectively. The ground part consists of the control station and the monitoring station, this system also includes 2 calibration stations in Hawaii and Australia. Its characteristic is: (1) release information about the GPS satellite ephemeris error correction, the satellite clock error correction and correction of ionospheric by the GEO satellite, (2) release the complete data of the GPS by GEO satellite. (3) GEO satellite navigation load transmit GPSL1 ranging signal. MSAS system can cover most areas of Chain from the point of view of satellite coverage range. The waters of China are covered by this system according to the ionospheric coverage range of the system. Therefore, carrying out differential positioning in the sea area of China by using the GPS receiver which adopts MSAS satellite-based enhanced mode, its signal can meet the needs of positioning.

\section{Experiment and analysis [4]}

The comparison test must be carried out for the GPS instrument which is set up on the known point, this is the requirement of specification, because only instruments that meet the accuracy requirements are allowed to be put into use.

\section{Comparison test [5]}

In order to test the accuracy of the analytical instrument, this test takes three different ways to make a comparison by using three sets of instruments, these three sets of instruments are Haida V8 RTK system, HD8500 DGPS system and TrimbleDSM232 DGPS system, its nominal accuracy is shown in Table 1.

Table 1: Nominal accuracy of instrument

\begin{tabular}{|c|l|}
\hline instrument classes & nominal accuracy \\
\hline V8 RTK & $\begin{array}{c}\text { RTK: plane } \pm 10 \mathrm{~mm}+1 \mathrm{ppm} \\
\text { altitude } \pm 20 \mathrm{~mm}+1 \mathrm{ppm}\end{array}$ \\
\hline HD8500 DGPS & differential beacon: plane static state $\leqslant 1 \mathrm{~m}$ \\
\hline TrimbleDSM232 DGPS & differential MSAS: plane static state $\leqslant 1 \mathrm{~m}$ \\
\hline
\end{tabular}

according to the technical specification, the comparison test of the above three instruments is carried out so as to test the static positioning accuracy of these instruments. V8 RTK system uses four known points to carry out parameter seeking and data comparison, the type of solution is narrow band fixed solution. HD8500 DGPS system and TrimbleDSM232 DGPS system carry out data acquisition through the station which is set up in the known point, the sampling interval time is three Seconds. Table 2 is the coordinate difference between the obtained recording point data and the coordinates of the known point by using the V8 RTK system, table 3 is the positioning accuracy and the coordinate difference by using HD8500 differential beacon and TrimbleDSM232 differential MSAS. 
Table 2: Comparison table of V8 RTK coordinate difference

\begin{tabular}{|c|c|c|c|}
\hline position point name & $\mathrm{dx}$ & $\mathrm{dy}$ & $\mathrm{dh}$ \\
\hline $\mathrm{G} 1$ & 0.034 & 0.032 & 0.013 \\
\hline $\mathrm{G} 2$ & 0.032 & 0.029 & 0.007 \\
\hline $\mathrm{G} 3$ & 0.019 & 0.012 & 0.008 \\
\hline $\mathrm{G} 4$ & 0.016 & 0.017 & 0.009 \\
\hline
\end{tabular}

Table 3: data comparison table between differential beacon and differential MSAS

\begin{tabular}{|c|c|c|c|}
\hline \multicolumn{2}{|c|}{ Instrument differential mode } & $\begin{array}{c}\text { HD8500 } \\
\text { differential beacon }\end{array}$ & $\begin{array}{c}\text { TrimbleDSM232 } \\
\text { differential MSAS }\end{array}$ \\
\hline \multirow{2}{*}{ positioning accuracy } & Ex & \pm 0.0225 & \pm 0.2002 \\
\cline { 2 - 4 } & Ey & \pm 0.0255 & \pm 0.5102 \\
\cline { 2 - 4 } & Eo & \pm 0.0341 & \pm 0.5481 \\
\hline coordinate difference & $\mathrm{dx}$ & -0.2379 & -0.2106 \\
\cline { 2 - 4 } & $\mathrm{dy}$ & 0.2327 & 0.2486 \\
\hline
\end{tabular}

Through the above test data, we can know that the maximum coordinate difference for V8 RTK is $\mathrm{dx}=0.034, \mathrm{dy}=0.032$, the coordinate difference for HD8500 differential beacon is $\mathrm{dx}=-0.2379$, $\mathrm{dy}=0.2327$, and the coordinate difference for TrimbleDSM232 differential MSAS is $\mathrm{dx}=-0.2106$, $d y=0.2486$. In the data comparison test, the positioning coordinate difference of the instrument used is within $1 \mathrm{~m}$. That is to say, the static positioning accuracy of the three differential methods is within $1 \mathrm{~m}$.

the HD8500 antenna, TrimbleDSM232 antenna and V8 RTK antenna is placed together and fixed on a certain position on a ship, the distance between each antenna phase center is about $10 \mathrm{~cm}$, the dynamic comparison test is carried out when the ship speed is about $6 \mathrm{kn}$. Because of the high accuracy of RTK, the difference between HD8500 differential beacon, TrimbleDSM232 differential MSAS and V8 RTK is mainly compared in this test. Compare the collected coordinates in the whole test, the biggest positioning difference between TrimbleDSM232 differential MSAS and V8 RTK is $2.6 \mathrm{~m}$, the biggest positioning difference between HD8500 differential beacon and V8 RTK is $1.5 \mathrm{~m}$, the positioning difference of these two methods is within $3 \mathrm{~m}$ during the data acquisition and comparison.

Accordingly the following specific test was carried out, we carried out 1: 25000 scale water depth survey in the ocean area, There are provisions in $<$ Specifications for hydrographic survey $>$ that the positioning error in map is less than $1 \mathrm{~mm}$, this is equivalent to the actual distance within $20 \mathrm{~m}$, the test area is more than 200 kilometers away from the coast. The test result is as follows: there is no differential GPS beacon signal at this time, GPS single point positioning can not meet the accuracy requirements, we use MSAS satellite-based differential model for measurement positioning, the instrument which we used is a TrimbleDSM232 receiver, the satellite differential signal is stable and normal in the whole measurement process, This model can be used to ensure the completion of the measurement task and its accuracy can meet the requirements of the specification.

\section{Conclusions}

This paper further confirmed the feasibility of the satellite-based wide area differential GPS positioning for the measurement of ocean water depth in Chinese waters through a data comparison test and application of the satellite-based differential measurement model, the emergence of this technology can further complement and improve the technology of wide area differential GPS, so that it can better meet the needs of users.

\section{References}

[1] Jiyu Liu. The principle and method of GPS satellite navigation and positioning [M] Beijing: Science Press, 2003. 
[2] GB12327-1998. Specifications for hydrographic survey [S]. Beijing: Standards Press of China, 1999.

[3] Jingnan Liu, Junyong Chen, Yanping Zhang. The principle and method of wide area differential GPS [M] .Beijing: Surveying and Mapping Press, 1999.

[4] Qin Zhang, Jiaquan Li. Principle and application of GPS measurement [M]. Beijing: Science Press, 2005.

[5] Jin Sang, Tiejun Zhang. Establishment and performance evaluation of RBN-DGPS system in coastal areas of China [J]. Bulletin of Surveying and Mapping, 1998, 17(2):56 58 .

[6] Rosenberg J. Schulzrinne H.Camarillo G.Johnston.A.Peterson , J.Sparks , and E.Schooler. SIP:Session Initiation Protocol. RFC 3261, June 2002. 